Case Report

\title{
3D-printed moulds facilitate making an anatomically matched cement spacer for reconstruction long bone defection after resection of tumors of humerous
}

\author{
Haitao Zhao ${ }^{1}$, Arun Sigdel ${ }^{2}$, Xizhi Hou ${ }^{1}$, Ting Zhang ${ }^{1}$, Guochuan Zhang ${ }^{1}$, Tao Sun ${ }^{1}$ \\ ${ }^{1}$ Department of Orthopedic Surgery, Third Hospital of Hebei Medical University, Shijiazhuang 050051, Hebei \\ Province, China. \\ ${ }^{2}$ Department of Surgical Oncology, Orthopedic Surgery unit, B.P. Koriala Memorial Cancer Hospital, \\ Bharatpur, Chitwan, Nepal.
}

\begin{abstract}
Background: A nail and cement spacer is one of the option for the reconstruction of the proximal humerus after tumor resection among prosthesis unaffordable patients. However, making the cement spacer anatomically match its replacement remains challenging.

Presentation of case: A 12-year-old boy was diagnosed with osteosarcoma in the right proximal humerus by core needle biopsy. After preoperative neo-adjuvant chemotherapy, a wide resection was performed, and the defect was reconstructed with an anatomically matched cement spacer. The cement spacer was fabricated using 3D-printed moulds, which were made according to the mirror image of the left humerus based on CT data. The post-operative course was uneventful, and at the 12-month follow-up, the patient is able to move with only some restriction in abduction and upward lift. The MSTD score was 21.

Conclusion: Fabrication of an anatomically matched cement spacer using 3D-printed moulds is a simple, inexpensive, and reproducible procedure for reconstruction complex bone defect.
\end{abstract}

Keywords: 3D-print; Anatomical cement spacer; Proximal humerus; Osteosarcoma

\section{Introduction}

Primary and metastatic tumors in the proximal humerus are relatively common and limb salvage surgery offers better function than amputation. $(1,2)$ Various techniques have been described for replacement of the proximal humerus after limb salvage. The options for reconstruction bone defect included osteoarticular allograft, allograft-prosthesis composite, freevascularized fibula graft, cement nail spacers, a sling procedure with a vascularized fibular graft, claviculo-pro-humerus and endoprosthetic replacement of the proximal humerus. (1, 3-8) Every reconstruction methods has its own advantages and disadvantages. Nail Cement spacers is an inexpensive and effective method, and especially suitable for indications of very small remaining distal stump of humeral, high risk of local infection, requirement of further adjuvant chemotherapy and radiotherapy.(9) Because every cement spacer was made by hand during the operation, the shapes of cement spacer were not anatomical and their surfaces were not so smooth.

During the last 5 years Computer-assisted design (CAD) and $\mathrm{CAD}$-rapid prototyping (CAD-RP) have developed well and been applied in orthopedic surgeries. The three dimensional (3D) printed guiding templates have been used for the placement screws of lumbar pedicle, C2 laminar, cervical pedicle, and thoracic pedicle.(1013) Recently, 3D-printed guiding templates have been developed to facilitate precise resection and accurate

\section{Correspondence}

Tao Sun M.D., PhD., Department of Orthopaedic surgery, Third Hospital of Hebei Medical University, 139 Ziqiang Road, Shijiazhuang 050051, Hebei Province, China. Tel: +86-0311-88602568; Fax: +86-311-87023626; E-mail: doctorsun@139.com 
reconstruction of malignant bone tumors. $(14,15)$ Even in a pilot study, 3D- printed calcaneal prosthesis has been applied in reconstruction after total calcanectomy. (16) Similar to endoprothesis, the application of 3D-printed prosthesis is limited by its high cost.

We describe a method to make an anatomically matched proximal humeral cement nail spacer using 3D-printed moulds in the resection and reconstruction of proximal humeral osteosarcoma.

\section{Presentation of case}

A 12-year-old boy presented with a two-month history of right shoulder pain. Radiographic studies revealed and abnormal lesion in the right proximal humerus (Fig 1), and thallium scintigraphy showed a high metabolic activity. Core needle biopsy confirmed the pathological diagnosis of osteosarcoma. At the time of diagnosis, no distant metastasis was detected. And the patient received neo-adjuvant chemotherapy using the appropriate treatment protocols.

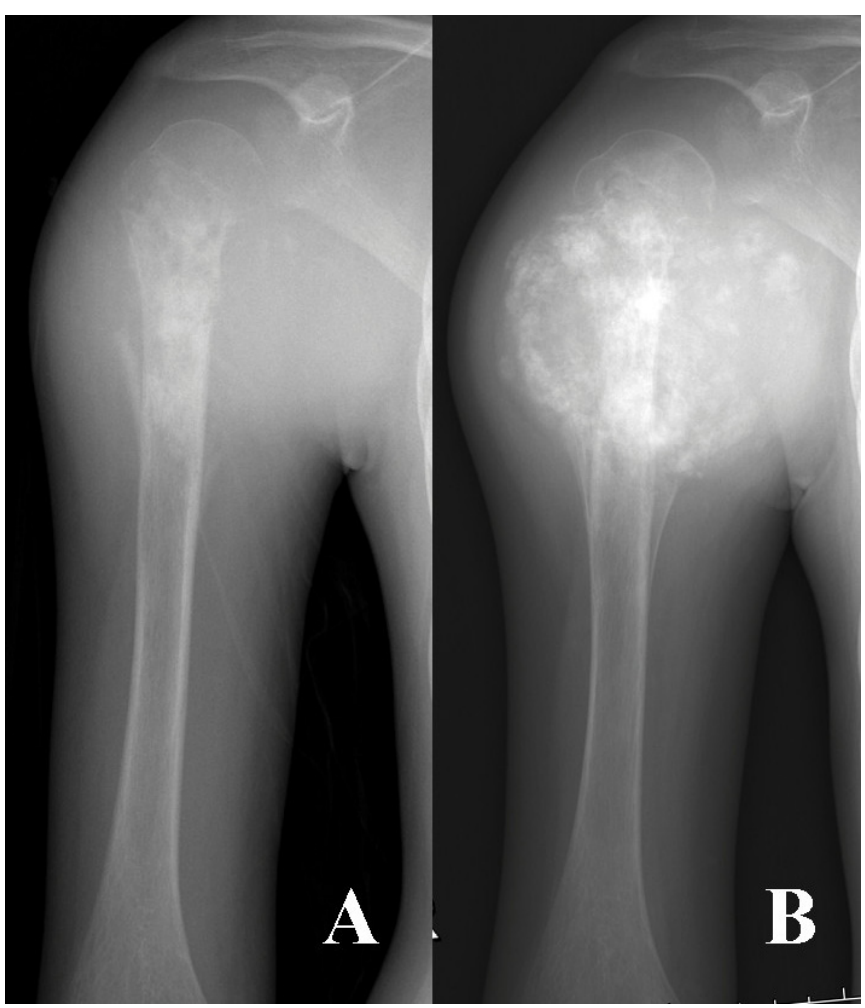

Fig 1 A plain radiograph shows a osteosarcoma in right proximal humerus at diagnosis (A). Another plain radiograph show the metaphyseal epicenter and extraosseous soft tissue component became calcification after chemotherapy before surgery (B).

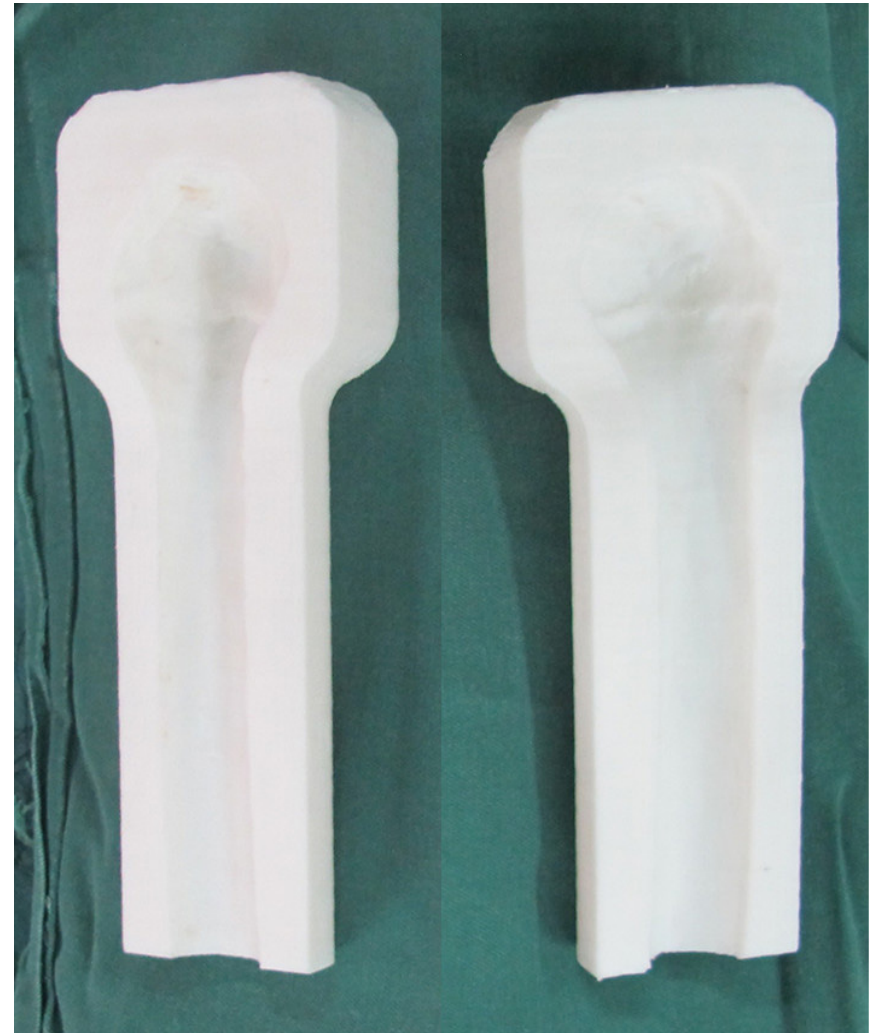

Fig 2 A photograph shows 3D-printed moulds, which were designed as two pieces in order to extrusion and separation the bone cement spacer easily when solid.

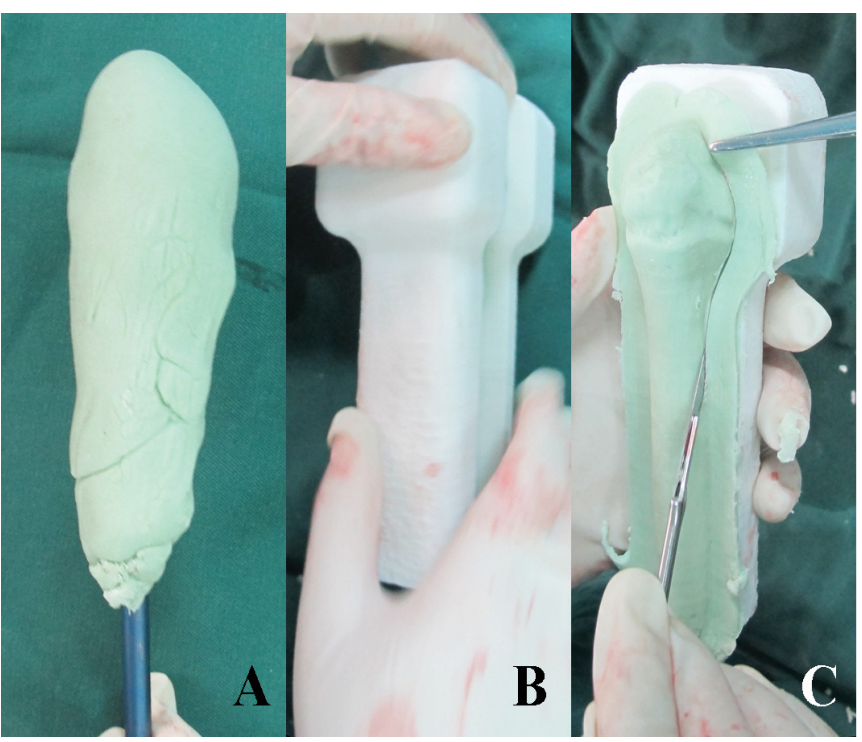

Fig. 3 Photographs show the manufacture procedure of cement spacer. The semisolid cement was moulded around the nail (A), and put between the two pieces of $3 \mathrm{D}$-printed moulds then press the moulds hard for shaping (B). The cement spacer was released from the moulds and the overflow was cut off and removed(C). 


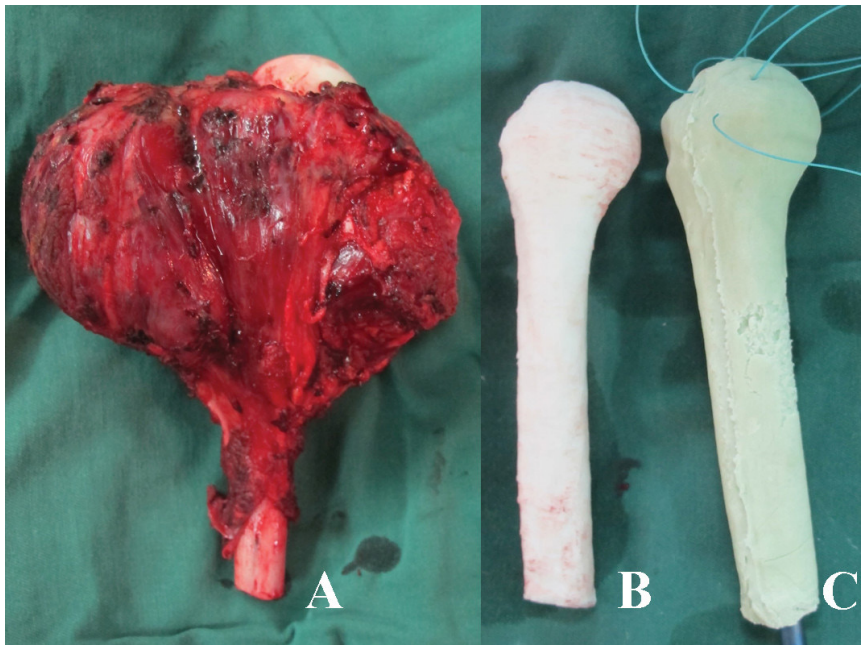

Fig 4 A photograph show the resected proximal humeral specimen with huge soft tissue mass (A). The $3 \mathrm{D}$-printed proximal humerus model was fabricated as a mirror image of left proximal humerus (B). The proximal humeral cement nail spacer is prepared ready for implantation with Non-absorbable No. 1 Ethibond sutures embedded (C).

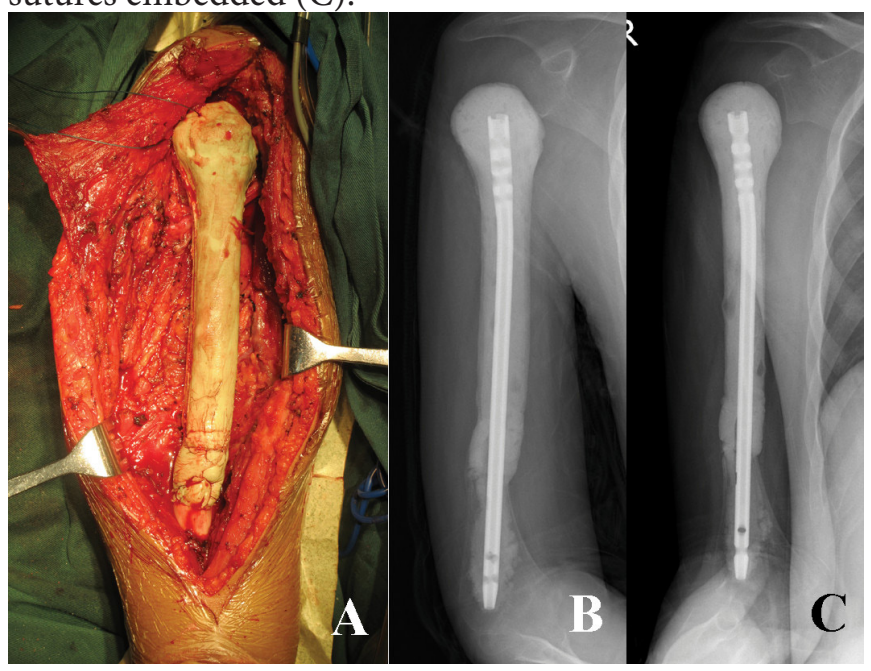

Fig 5 A photographs show the implantation and fixation the nail cement spacer into the residue distal humeral medullar cavity (A). Post-operative radiographs show an anatomically matched cement spacer with a smooth surface of proximal humerus (B and C).

The wide resection of proximal humeral osteosarcoma followed by an anatomically matched cement nail spacer reconstruction was performed. The anatomically matched cement spacer was made with facilitated of $3 \mathrm{D}$-printed moulds, which is according to the mirror image of the left proximal humerus based on lelical CT DICOM data. The mould was fabricated by $3 \mathrm{D}$ printing of the commercial (Waston 3D-ORTHO, China) using polylactic acid (PLA) $1.75 \mathrm{~mm}$ white (Fig.2). The $3 \mathrm{D}$-printed mould was sterilized by Ethylene oxide fumigation method before surgery (Fig 2).

The patient was positioned supine on an operating table under general anesthesia. The lesion was approached by way of an extended deltopectoral anterolateral incision. Previous biopsy tracts were incorporated into the incision and were completely excised. The lesion was resected transarticularly, and the glenohumeral joint was disarticulated by dividing the long head of biceps as well as the tendinous portion of the rotator cuff. The tendons of pectoralis major, latissimus dorsi, teres major and long head of biceps were detached. A cuff of normal soft tissue was retained around the proximal humerus. Meticulous dissection was performed, and an intraarticular proximal humerus with the humeral diaphysis was isolated at $5 \mathrm{~cm}$ from the most distal part of the lesion (as determined by MRI) and cut with a wire saw. Marrow from remaining distal humeral diaphysis was sent for frozen section evaluation. The proximal humeral tumor with huge soft tissue mass was removed, and hemostasis was performed immediately after that. The humeral canal was reamed to accept the intramedullary nail. Figure 3 showed the procedure of making the bone cement spacer. A nail antibiotic cement spacer was prepared depending on the length of humerus resected. When became semisolid, bone cement was patted around the nail as shown in Fig $3 \mathrm{~A}$. Then the nail cement spacer was put between two pieces of 3D-printed moulds, and extruded for shaping (Fig 3B). One piece of mould was removed and excess cement cleaned up carefully (Fig 3C). Six non-absorbable No. 1 Ethibond sutures were sewed through the cement head before bone cement became solid (Fig 4C). The distal end of nail was inserted into the reamed intramedullary canal filled with cement (Fig 5A). The cement head was abutted into the glenoid. Soft tissue was crossed suture and reattachment of the residual muscles around the shoulder girdle to provide static stability. The residual muscles were sutured around the cement nail spacer; soft tissue and skin were sutured over a negative suction drain. Post-operative radiograph showed the perfect fit of anatomically matched cement nail spacer (Fig 5).

Post-operative operative course was uneventful. The arm was placed in an arm chest bandage after surgery. Stitches were removed after two weeks, and the hand, wrist and elbow were mobilized. After six to eight weeks, the sling was removed and passive mobilization started. Chemotherapy was 
carried out at four week after surgery using the appropriate treatment protocols. In four month after surgery, the patient was able to perform daily activities such as hand and face washing, eating, lifting a cup and other household works. Ranges of motion of the right shoulder remained restricted mainly in abduction and upward lift, with $15^{\circ}$ abduction, $10^{\circ}$ of upward lift and $10^{\circ}$ of both abduction and upward lift. The Musculoskeletal Tumor Society (MSTS) functional score for this patient was 21 points.

\section{Discussion}

Limb salvage surgery for the proximal humeral tumors rather than amputation has become a treatment choice during last few decades because the introduction of effective neoadjuvant chemotherapy. $(3,5,7,8)$ Although a salvaged flail shoulder happens in most patients, limb salvage surgery still offers functional, cosmetic, and emotional advantages. $(7,9)$ Endo-prothesis is the most common mode of reconstruction nowadays, but its high cost (more than 2,000 US \$) is the major limiting factor in many parts of the world.(9) Because most replacements of the proximal humerus act as functional spacers rather than as an articulating reconstruction, a nail cement spacer has been used as an inexpensive alternation (the implant with cementation costs less than US \$100)) in proximal humeral reconstruction.(9) Although a nail cement spacer has its advantages, it is not a standard procedure because it has narrow time window of moulding and is made by hand with individual difference. With the help of CAD-RP technique, we developed a reproducible method to make an anatomically matched cement spacer.

We are trying to develop a standard procedure of making an anatomically matched cement spacer. First, a proximal humerus model was fabricated as a mirror image of the contralateral side of bone. Second, two pieces of moulds were fabricated according to the humerus model which is the most anatomical. It took about 8 hours to make the 3D-printed moulds and proximal humerus model, and the cost was about $\$ 30$, which is similar to previous report. (15)There are four useful tips in the operation. Firstly, moulding the cement on the nail as vertical oval in cross section can help the fully filling of the cement when closing the moulds. Secondly, spraying sterile saline on the inner surfaces of 3D-printed moulds can make releasing easily and cement surface smoothly. Thirdly, the upper piece of mould can be removed a little early, and the overflow cement can be cleaned up and sutures can be sewed into cement as soon as possible. Theses sutures make the soft tissue reconstruction much easier. And finally, when cement spacer becomes completely solid, it can be used in the same way as prosthesis. In a word, it is a simple, inexpensive, and reproducible produce to make an anatomically matched cement spacer using 3D-printed moulds.

The cement spacers made by method has several advantages. First of all, it is anatomical and match exactly in the space. Combining with 3D-printed guiding templates, it can achieve precise resection and reconstruction of bone tumor anatomically. Secondly, the cement spacer was integral moulding, which may result in better structure and smoother surface. In this case, we use an intra-medullary nail to connect the cement spacer and remaining bone. However, if a plate and nails are use to connect the cement spacer and bone, a groove for plate and nails in the 3D-printed mould should be planned and reserved. And then, a locking plate is recommended. This plate cement spacer has potential use in distal radius reconstruction after wide resection. Furthermore, the plate cement spacer combine with single condyle surface replacement may be used in single condyle reconstruction of distal humerus and proximal tibia when tumor involves articular surface.

Anatomical matched implant or prosthesis are likely to be safe and can achieve good functions. Antibioticimpregnated cement spacer is widely used in the treatment of infection associated total hip of knee arthoplasty.(17-19) A simple, inexpensive spacer is basically required to keep the tissue planes intact and prevents soft tissue contraction during the interpretative period of 2-stage revision. During the operation, the surgeons tried their best to fabricate the cement spacers like the replaced bones. With the help of $3 \mathrm{D}$-prineted moulds, any surgeon can make an ideal spacer, which is anatomically matched, antibiotic-impregnated, and with antibiotic sutures embedded

The limitation of our report is the relatively short of follow-up time. However, we can report that this method is a simple, cost-effective technique without more complication. Second, the potential benefits of this anatomically matched cement spacer is in improving the reconstruction of bone defect and soft tissue but may not help reduce the risk of local recurrence and metastasis.

\section{Conclusion}

We reported the first cases of 3D-printed moulds 
facilitated making an anatomically matched cement spacer for reconstruction of proximal humerus. Shortterm clinical outcomes were satisfactory. This method can be a promising option for making a cement spacer anatomically matched.

\section{Acknowledgements}

We acknowledge Xiaodong Cheng for their assistance and cooperation in this study.

\section{References:}

1. Marcove RC, Lewis MM, Huvos AG. En bloc upper humeral interscapulo-thoracic resection. The Tikhoff-Linberg procedure. Clinical Orthopaedics \& Related Research. 1977;124(124):219.

2. Sissons HA. Bone Tumors. General Aspects and Data on 6211 Cases. Journal of Clinical Pathology. 1979;32(7).

3. Frassica FJ, Sim FH, Chao EY. Primary malignant bone tumors of the shoulder girdle: surgical technique of resection and reconstruction. American Surgeon. 1987;53(5):264-9.

4. Gebhardt MC, Roth YF, Mankin HJ. Osteoarticular allografts for reconstruction in the proximal part of the humerus after excision of a musculoskeletal tumour. J Bone Joint Surg [Am]. Journal of Bone \& Joint Surgery. 1990;72(3):334-45.

5. Wada T, Usui $M$, Isu $K$, Yamawakii S, Ishii S. Reconstruction and limb salvage after resection for malignant bone tumour of the proximal humerus. A sling procedure using a free vascularised fibular graft. Journal of Bone \& Joint Surgery British Volume. 1999;81(5):808-13.

6. Hsu RW, Wood MB, Sim FH, Chao EY. Free vascularised fibular grafting for reconstruction after tumour resection. 1997;79(1):36-42.

7. Rödl RW, Gosheger G, Gebert C, Lindner N, Ozaki T, Winkelmann W. Reconstruction of the proximal humerus after wide resection of tumours. Journal of Bone \& Joint Surgery British Volume. 2002;84(7):1004.

8. O'Connor MI, Sim FH, Chao EY. Limb salvage for neoplasms of the shoulder girdle. Intermediate reconstructive and functional results. Journal of Bone \& Joint Surgery. 1997;17(12):1872.

9. Kundu ZS, Gogna P, Gupta V, Kamboj P, Singla R, Sangwan SS. Proximal humeral reconstruction using nail cement spacer in primary and metastatic tumours of proximal humerus. Strategies in Trauma and Limb Reconstruction. 2013;8(3):14954.

10. Lu S, Xu YQ, Lu WW, Ni GX, Li YB, Shi JH, et al. A novel patient-specific navigational template for cervical pedicle screw placement. Spine. 2009;34(26):959-66.

11. Lu S, Xu YQ, Zhang YZ, Li YB, Xie L, Shi JH, et al. A novel computer-assisted drill guide template for lumbar pedicle screw placement: a cadaveric and clinical study. International Journal of Medical Robotics \& Computer Assisted Surgery. 2009;5(2):184-91.

12. Lu S, Xu YQ, Zhang YZ, Xie L, Guo H, Li DP. A novel computer-assisted drill guide template for placement of C2 laminar screws. European Spine Journal. 2009;18(9):1379-85.

13. Ma T, Xu YQ, Cheng YB, Jiang MY, Xu XM, Xie $\mathrm{L}$, et al. A novel computer-assisted drill guide template for thoracic pedicle screw placement: a cadaveric study. Archives of Orthopaedic and Trauma Surgery. 2012;132(1):65-72.

14. Zhang Y, Yin QS, Huang HY. Surgical treatment of pelvic malignant tumors with individualized hemi-pelvic. Orthopedic Journal of China. 2009;17(3):190-2.

15. Ma L, Zhou Y, Zhu Y, Lin Z, Wang Y, Zhang Y, et al. 3D-printed guiding templates for improved osteosarcoma resection. Scientific Reports. 2016;6:23335.

16. Imanishi J. Three-dimensional printed calcaneal prosthesis following total calcanectomy. International Journal of Surgery Case Reports. 2015;10:83-7.

17. Cui Q, Mihalko WM, Shields JS, Ries M, Saleh KJ. Antibiotic-Impregnated Cement Spacers for the Treatment of Infection Associated with Total Hip or Knee Arthroplasty. 2007;89(4):871-82.

18. Durbhakula SM, Czajka J, Fuchs MD, Uhl RL. Antibiotic-loaded articulating cement spacer in the 2-stage exchange of infected total knee arthroplasty. Journal of Arthroplasty. 2004;19(6):768-74.

19. Hsu YC, Cheng HC, Ng TP, Chiu KY. Antibioticloaded cement articulating spacer for 2-stage reimplantation in infected total knee arthroplasty: a simple and economic method. Journal of Arthroplasty. 2007;22(7):1060-6. 